\title{
VISUALIZAÇÃO DO CONHECIMENTO: UMA TÉCNICA PARA APRIMORAMENTO NA PRODUÇÃO DE OBJETOS DE APRENDIZAGEM NA EDUCAÇÃO
}

\section{VISUALIZATION OF KNOWLEDGE: A TECHNIQUE FOR ENHANCEMENT IN THE PRODUCTION OF LEARNING OBJECTS IN EDUCATION}

\author{
Renan de Paula Binda, Mestrando \\ Monica Dos Santos Mondo \\ Luciane Fadel, Doutora \\ Vânia Ribas Ulbricht' Doutora
}

(1) Universidade Federal de Santa Catarina (UFSC) e-mail: renanbinda1@gmail.com

(2) Universidade Federal de Santa Catarina (UFSC) e-mail: monicamondo87@gmail.com

(3) Universidade Federal de Santa Catarina (UFSC) e-mail: liefadel@gmail.com

(4) Universidade Federal de Santa Catarina (UFSC) e-mail: vrulbricht@gmail.com

Visualização do Conhecimento; Objetos de aprendizagem; educação;

O presente artigo apresenta uma revisão sistemática sobre a Visualização do Conhecimento como potencial ferramenta para auxilio no desenvolvimento de objetos de aprendizagem na EaD. Essa pesquisa teve como objetivo verificar a existência de diretrizes ou modelos capazes de oferecer suporte à tradução do conhecimento. De modo geral, a análise permitiu conhecer algumas aplicações da visualização do conhecimento aplicado a área da educação, constatando carência de ferramentas capazes de oferecer suporte ao desenvolvimento de objetos de aprendizagem. 


\section{$16^{\circ}$ \\ ERGODESIGN USIHC CINAHPA}

$16^{\circ}$ Ergodesign - Congresso Internacional de Ergonomia e Usabilidade de Interfaces Humano Tecnológica: Produto, Informações Ambientes Construídos e Transporte

$16^{\circ}$ USIHC - Congresso Internacional de Ergonomia e Usabilidade de Interfaces Humano Computador

CINAHPA | 2017 - Congresso Internacional de Ambientes Hipermídia para Aprendizagem.

\author{
Visualization of Knowledge; Learning objects; education;
}

This article presents a systematic review on Visualization of Knowledge as a potential tool to assist in the development of learning objects in Education. This research aimed to verify the existence of guidelines or models capable of supporting the translation of the knowledge to be disseminated. In general, the analysis allowed to know some applications of the tool in the area of education, noting the lack of tools capable of meeting this need.

\section{Introdução}

A modalidade de ensino a distância surge como estratégia da educação continuada e para atender demandas sociais distante do acesso à educação tradicional. Com a evolução das novas Tecnologias da Informação e Comunicação (TIC's), a EaD tem explorado os meios digitais para disseminar conhecimento.

As TIC's vêm proporcionando novas expectativas de apropriação e construção de conhecimento, contribuindo para a capacitação de cidadãos. A Educação a Distância, conforme Decreto 5.622, de 19.12.2005, é a modalidade educacional na qual a mediação didático-pedagógica nos processos de ensino e aprendizagem ocorre com a utilização de meios e tecnologias de informação e comunicação (TIC's), com estudantes e professores desenvolvendo atividades educativas em lugares ou tempos diversos.

As estratégias utilizadas nas aulas a distância vêm sendo transmitidas nos moldes tradicionais, com o surgimento de novas ferramentas que suportam o ensino-aprendizado novas formas de aprender podem ser estimuladas. Silva (2003, p.50) salienta que essa nova forma de aprender envolve tipos específicos de percepção e de cognição do sujeito, demandando, por isso mesmo, a superação de formas tradicionais de aprendizagem pela recuperação, reinserção e dinamização das dimensões interativas e lúdicas do processo.

Segundo o Censo EaD de 2016: Conforme opinião dos participantes, a EAD exige inovação tecnológica e administrativa, infraestrutura tecnológica e de apoio ao aluno em níveis mais elevados quando em comparação à modalidade educacional presencial. As taxas de evasão reportadas nos cursos a distância são maiores que as nos cursos presenciais (os cursos regulamentados totalmente a distância apresentam os índices mais altos).

Os objetos de aprendizagem (OA) na EaD vêm oferecer suporte às necessidades de sistemas flexíveis e adaptativos nos meios educacionais e de difusão do conhecimento. Segundo o Institute of Eletrical and Eletronics Engineers-IEEE (2002) OA são qualquer entidade, digital ou não, que pode ser utilizada, reutilizada ou referenciada durante a aprendizagem apoiada pela tecnologia.

Fatores como a não interação com outros alunos e a falta de suporte do professor, deposita nos objetos de aprendizagem uma responsabilidade ainda maior de cativar e engajar o aluno. Meios para diminuir a sobrecarga cognitiva e a necessidade de oferecer materiais educacionais mais atrativos e de fácil compreensão se faz cada dia mais forte.

As formas visuais utilizam imagens revestidas conceitualmente para, a partir de Wang e Jacobson (2011), auxiliar pessoas a construir e transmitir introspecções, pensamentos, visando melhorar a compreensão e comunicação. Formatos gráficos, desenhos, diagramas, metáforas visuais, segundo Eppler e Burkhard (2007), são exemplos de visualização do conhecimento, que usados como dispositivos de comunicação desencadeiam atividades sensoriais que motivam observadores a reconstruir significados.

Por isso, a relevância desta pesquisa justifica-se pela intenção de organizar e representar visualmente o conhecimento, traduzindo para formato de objetos de aprendizagem. Para, dessa forma, torna-los mais próximo da realidade dos alunos dos cursos oferecidos na modalidade a distância, estimando 


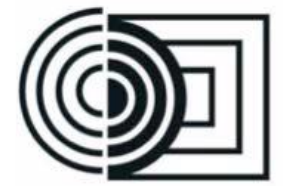

\section{$16^{\circ}$}

ERGODESIGN USIHC CINAHPA

melhorias na forma de ensino e principalmente para gerar maior engajamento do aprendiz.

\section{Visualização do Conhecimento (VC)}

Como instrumento, a VC, explora nosso potencial inato de processar representações visuais para tarefas intensivas em conhecimento (BURKHARD; MEIER, 2004), além da capacidade de obter sobre o usuário um efeito emocional em termos de engajamento e motivação, (TERGAN et al., 2005).

Tornando-se um campo de estudo investigativo dos formatos visuais apoiado em processos meta-cognitivos estimula o pensamento de alto nível, auxilia pessoas a desenvolver uma consciência de suas representações mentais, favorecendo a geração, representação, estruturação, recuperação, compartilhamento e uso do conhecimento, (BURKHARD; MEIER, 2005; JACOBSON; WANG, 2004; TERGAN et al., 2006).

A VC é fruto da integração de pesquisas de áreas como a visualização da informação, psicologia cognitiva, arte, design, ciências da comunicação, arquitetura da informação e gestão do conhecimento (BURKHARD; MEIER, 2004). $16^{\circ}$ Ergodesign - Congresso Internacional de Ergonomia e Usabilidade de Interfaces Humano Tecnológica: Produto, Informações Ambientes Construídos e Transporte

$16^{\circ}$ USIHC - Congresso Internacional de Ergonomia e Usabilidade de Interfaces Humano Computador

CINAHPA | 2017 - Congresso Internacional de Ambientes Hipermídia para Aprendizagem.

\section{Procedimentos Metodológicos da RSL}

Figura 4: Procedimentos metodológicos

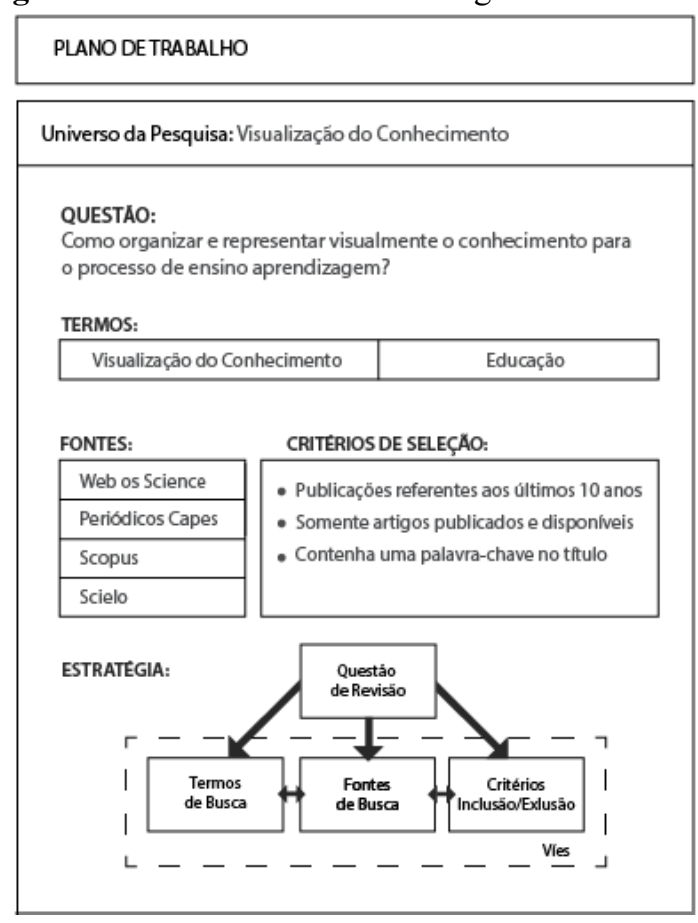

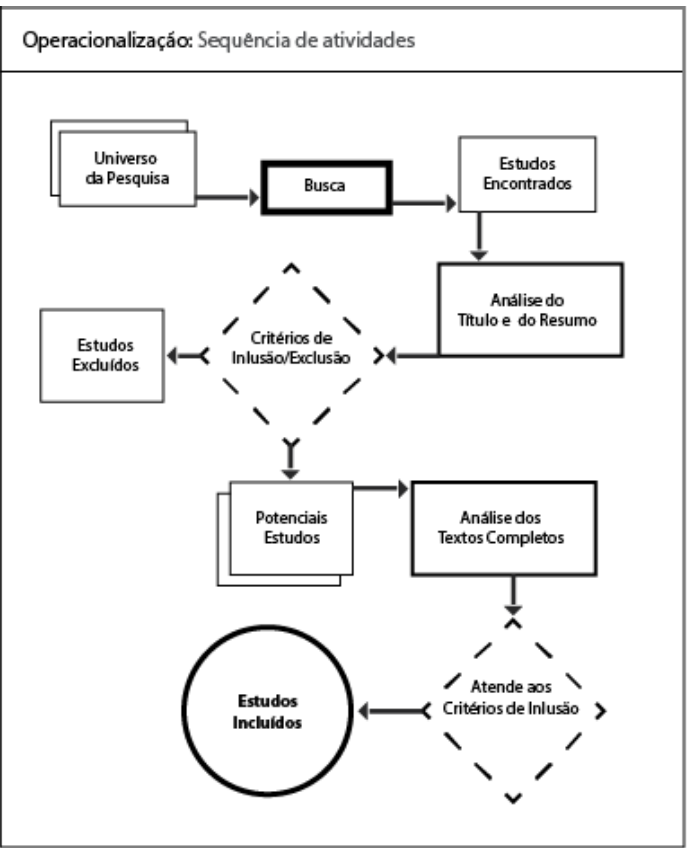

Fonte: Elaborado pelos autores a partir de Dresh et al. (2015)

O método de pesquisa utilizado para esta revisão sistemática da literatura apoia-se na Design 


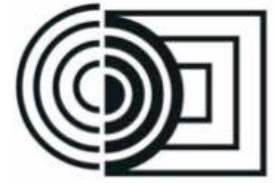
CINAHPA

Science Research (DSR), que segundo Dresch et al. (2015), tem por objetivo formar um arcabouço teórico-prático dos artefatos utilizados para a solução de um determinado problema. Escolhemos este método pois conta com um procedimento claro e alinhado aos objetivos do trabalho.

A RSL, nesta pesquisa, teve por objetivo conhecer os conceitos adotados referentes a VC aplicados ao processo de ensino-aprendizagem e como este tema vem sendo abordado pela comunidade acadêmica. A estratégia elaborada e as etapas da sequência de atividades adotadas encontram-se inseridos nos procedimentos metodológicos esquematizado no plano de trabalho, como pode ser observado na Figura 4.

No plano encontra-se a questão de pesquisa, como também os termos adotados para a revisão. Abaixo apresentamos o termo usado para a busca nas bases de dados.

(TITLE (knowledge AND visualization) AND TITLEABS-KEY (education) AND DOCTYPE (ar) AND PUBYEAR > 2004

A seleção das bases de dados considerou a disponibilidade de recursos e fonte de busca usuais e abrangentes. A seguir apresentaremos as bases de dados eletrônicas utilizadas.

\section{Resultados}

Nesta seção são apresentados os resultados da busca e análise dos artigos, segundo os procedimentos seguidos para a RSL. As bases de dados eletrônicas utilizadas foram, Web of Science, Periódicos CAPES, SCOPUS e SCIELO por permitirem acesso a um número diversificado de conteúdo.

\subsection{Busca dos artigos}

A Revisão Sistemática da Literatura foi realizada nos dias 23, 24, 25 de janeiro do ano de 2017, retornando 62 estudos no total (Tabela 1). $16^{\circ}$ Ergodesign - Congresso Internacional de Ergonomia e Usabilidade de Interfaces Humano Tecnológica: Produto, Informações Ambientes Construídos e Transporte

$16^{\circ}$ USIHC - Congresso Internacional de Ergonomia e Usabilidade de Interfaces Humano Computador

CINAHPA | 2017 - Congresso Internacional de Ambientes Hipermídia para Aprendizagem.

Tabela 1: Localização e seleção dos estudos

\begin{tabular}{|l|l|l|}
\hline Base de Dados & Localizados & Selecionados \\
\hline Web of Science & 21 & 1 \\
\hline Capes & 30 & 7 \\
\hline Scopus & 11 & 2 \\
\hline Scielo & 0 & 0 \\
\hline
\end{tabular}

Fonte: Elaborado pelos autores

Os critérios citados no plano de trabalho nortearam a triagem dos artigos resultando na exclusão de arquivos em duplicidade e seleção dos que se encontravam disponíveis para acesso. Como também a leitura preliminar dos resumos apresentados pelos artigos.

\subsection{Análise dos artigos}

A seleção contou com nove artigos como potenciais estudos. No Quadro 1 apresentamos os títulos dos nove potenciais estudos selecionados para análise dos textos completos.

Quadro 1: Artigos selecionados

\begin{tabular}{|c|l|}
\hline 1 & $\begin{array}{l}\text { Ifenthaler, D. AKOVIA: Automated } \\
\text { Knowledge Visualization and Assessment. } \\
\text { Tech Know Learn, 2014. }\end{array}$ \\
\hline 2 & $\begin{array}{l}\text { Chen, M. Data, Information, and Knowledge in } \\
\text { Visualization. IEEE Computer Society, 2009. }\end{array}$ \\
\hline 3 & $\begin{array}{l}\text { Coffey, J. W. Hoffman, R. Cañas, A. Concept } \\
\text { map-based knowledge modeling: perspectives } \\
\text { from information and knowledge } \\
\text { visualization. Palgrave Macmillan Ltd. 2006. }\end{array}$ \\
\hline 4 & $\begin{array}{l}\text { Sasieta, H. A. M. Beppler, F. B. Pacheco, R. C. S. } \\
\text { Um modelo para a visualização do } \\
\text { conhecimento baseado em arquétipos visuais. } \\
\text { Acta Scientiarum Technology: Maringá, 2012. }\end{array}$ \\
\hline 5 & $\begin{array}{l}\text { Wang, M. Peng, J. Cheng, B. Zhou, H. Liu, J. } \\
\text { Knowledge Visualization for Self-Regulated } \\
\text { Learning. Educational Technology \& Society, 2014. }\end{array}$ \\
\hline 6 & $\begin{array}{l}\text { Wang, Y-H. Liao, H-C. Promoting English } \\
\text { Oral Communication and Higherorder } \\
\text { Thinking in Taiwanese esl Students Through } \\
\text { the Use of Knowledge Visualization } \\
\text { Techniques. Perceptual \& Motor Skills: } \\
\text { Learning \& Memory, 2014. }\end{array}$ \\
\hline
\end{tabular}




\section{$16^{\circ}$ \\ ERGODESIGN USIHC CINAHPA}

$16^{\circ}$ Ergodesign - Congresso Internacional de Ergonomia e Usabilidade de Interfaces Humano Tecnológica: Produto, Informações Ambientes Construídos e Transporte

$16^{\circ}$ USIHC - Congresso Internacional de Ergonomia e Usabilidade de Interfaces Humano Computador

CINAHPA | 2017 - Congresso Internacional de Ambientes Hipermídia para Aprendizagem.
7 Guangzheng Lia, Xingang Songa. New Visualization-oriented Knowledge Service Platform. SciVerse ScienceDirect, Procedia Engineering 15, 2011.

8 Kabanova1, N. N., Pankova, N. M., Hollenbeck, J. E., Prokhorova, K. E.. Challenges of scientific knowledge visualization in publication productivity of the university academic staff. SHS Web of Conferences 28, 2016.

9 Wang, M., Wu, B., Kinshuk, Chen N-S., Spector, J. M. Connecting problem-solving and knowledge-construction processes in a visualization-based learning environment. Computers \& Education 68, 2013.

Fonte: Elaborado pelos autores

Abaixo listamos os nove potenciais estudos com seus objetivos de pesquisa, a metodologia abordada e suas respectivas conclusões.

\section{Artigo 1:}

- Objetivo: Trabalho teórico procura revisar a ferramenta AKOVIA, baseada na web que analisa representações de linguagem natural e gráfica do conhecimento.

- Conclusão: Os resultados do teste validaram a ferramenta, pois a ferramenta permitiu que os alunos compreendessem melhor o fluxo textual e identificar conceitos ausentes ou mesmo equivocados.

\section{Artigo 2:}

- Objetivo: Estudo teórico analisa o papel atual e futuro da informação e do conhecimento no desenvolvimento de tecnologia de visualização.

- Conclusão: A visualização prosperou ao ajudar usuários a transferir conhecimento do espaço computacional para o espaço perceptivo e cognitivo. Aponta-se a necessidade de infraestrutura, como métodos e ferramentas, para recolher dados sobre os processos de visualização, e promover melhorias.

\section{Artigo 3:}

- Objetivo: Trabalho teórico explora a ideia de modelagem do conhecimento procurando definila como ferramenta capaz de ilustrar domínios através de mapas conceituais.

- Conclusão: O artigo conclui com uma discussão sobre maneiras específicas em que as abordagens de modelagem de conhecimento podem ser empregadas para criar, apresentar e organizar os recursos eficazes de aprendizagem eletrônica.

\section{Artigo 4:}

- Objetivo: Propor modelo para facilitar a visualização do conhecimento armazenado em repositórios digitais usando arquétipos visuais. Trabalho teórico analisa representações visuais.

- Conclusão: Utilizar arquétipos nas representações visuais facilita a divulgação do conhecimento já que ao fazer parte da visão do mundo dos usuários, podem facilmente ser associadas a conhecimentos prévios.

\section{Artigo 5:}

- Objetivo: Propor a VC como abordagem para resolver o problema da sobrecarga cognitiva de aprendizes que utilizam a web para autoaprendizagem. Realiza uma avaliação e aplica entrevistas com estudantes para medir a satisfação e aceitação de um sistema baseado na VC.

- Conclusão: O resultado da avaliação reflete o sucesso do sistema em termos de satisfação do usuário, aceitação e efetividade no aprendizado.

\section{Artigo 6:}

- Objetivo: O estudo examinou estudantes que usam mapas conceituais em um curso Freshman English. $\mathrm{O}$ experimento qualitativo analisa o curso de inglês como estudo de caso.

- Conclusão: Os alunos que utilizaram mapas conceituais apresentaram desempenho significativamente melhor em todos os aspectos comparados ao grupo de alunos que utilizou métodos tradicionais. Para pesquisas futuras os autores sugerem averiguar como o uso de mapas conceituais é capaz de reduzir a ansiedade de estudantes.

\section{Artigo 7:}

- Objetivo: Apresentar um protótipo de serviço de 


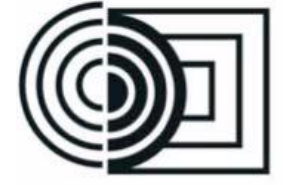
CINAHPA

conhecimento orientada para a visualização do conhecimento na forma de plataforma visando a transferência de experiências.

- Conclusão: A plataforma orienta de maneira eficaz criar sites cuja navegação, organização, rotulagem e pesquisa possam ser feitas de forma satisfatória e eficiente.

\section{Artigo 8:}

- Objetivo: O estudo procurou de forma teórica analisar os desafios relacionados a percepção de informação.

- Conclusão: Afirma que a tecnologia da informação permite através dos métodos de visualização a comunicação e promoção dos resultados científicos efetivando o reconhecimento desse conhecimento pela sociedade.

\section{Artigo 9:}

- Objetivo: Abordar o desafio de conectar o processo de construção de conhecimento com o problem-solving. Utilizando ferramentas cognitivas realiza experiência com participantes para analisar um ambiente de aprendizagem baseado em visualização.

- Conclusão: O ambiente contribui para a aprendizagem baseada em problemas em domínios complexos auxiliando alunos na conexão e articulação do conhecimento explorando problemas para construir novas soluções.

\subsection{Avaliação da Qualidade}

Os estudos selecionados foram avaliados de forma qualitativa. Para a avaliação utilizamos três dimensões, a partir de Harden; Gough, (2012): qualidade da execução do estudo, adequação à questão de revisão, adequação ao foco da revisão. A qualidade da revisão refere-se a forma como o estudo foi conduzido, analisando se o mesmo se encontra dentro dos padrões científicos considerados adequado. Também é averiguado se o estudo está apoiado em fatos e dados.

As atribuições para a análise variam entre baixa, $16^{\circ}$ Ergodesign - Congresso Internacional de Ergonomia e Usabilidade de Interfaces Humano Tecnológica: Produto, Informações Ambientes Construídos e Transporte

$16^{\circ}$ USIHC - Congresso Internacional de Ergonomia e Usabilidade de Interfaces Humano Computador

CINAHPA | 2017 - Congresso Internacional de Ambientes Hipermídia para Aprendizagem.

média e alta. Alta para os artigos que atende os requisitos científicos e que está em contexto idêntico ao definido nesta revisão. A atribuição média foi concedida aqueles estudos que não atendem totalmente aos requisitos e que se encontra em contexto semelhante aos desta revisão, e baixa para aqueles que não se adequam e se inserem em contexto diverso.

Quadro 2: Avaliação da Qualidade

\section{Avaliação da Qualidade dos Estudos}

\begin{tabular}{|c|c|c|c|}
\hline Estudos & $\begin{array}{c}\text { Qualidade da } \\
\text { Execução }\end{array}$ & $\begin{array}{c}\text { Adequação à } \\
\text { questão }\end{array}$ & $\begin{array}{c}\text { Adequação ao } \\
\text { foco }\end{array}$ \\
\hline $\mathbf{1}$ & Alta & Baixa & Alta \\
\hline $\mathbf{2}$ & Alta & Média & Média \\
\hline $\mathbf{3}$ & Alta & Alta & Alta \\
\hline $\mathbf{4}$ & Alta & Alta & Alta \\
\hline $\mathbf{5}$ & Alta & Média & Média \\
\hline 6 & Alta & Alta & Alta \\
\hline 7 & Alta & Média & Alta \\
\hline $\mathbf{8}$ & Alta & Alta & Alta \\
\hline $\mathbf{9}$ & Alta & Média & Alta \\
\hline
\end{tabular}

Fonte: Elaborado pelos autores

\subsection{Síntese da RSL}

A visualização do Conhecimento vem sendo aplicada, conforme revelou nossa pesquisa, em diferentes âmbitos. Na área da educação, essa técnica tem sido utilizada em contextos que objetivam melhorar a compreensão dos alunos e resolver o problema da sobrecarga cognitiva, além de facilitar o acesso a grandes quantidades de informação.

A maior parte dos artigos analisados focaram seu objetivo em promover a transferência de conhecimento, facilitar o acesso e compreensão de conhecimento e diminuição da sobrecarga cognitiva. Os artigos não apresentam convergência nos objetivos, abordam temas diferentes como, a proposta de solução para sobrecarga cognitiva (Wang, 2014), mudanças no processo educativo (Kabanova1, 2016), otimizar critérios de avaliação automatizada (Ifenthaler, 2014), entre outros.

As pesquisas em sua maioria são teóricas. Apenas três dos nove trabalhos apresentavam estudo de caso. Os estudos empíricos apresentaram resultados qualitativos satisfatórios com a
Realização:
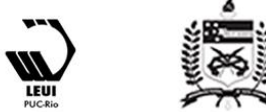


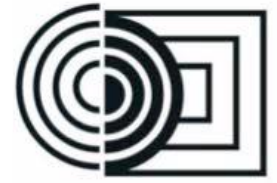
CINAHPA

aplicação da VC para solucionar problemas relacionados à aprendizagem. Os trabalhos teóricos contribuíram com analises e revisões sobre a VC e sua aplicação.

Nas conclusões, os artigos apresentam resultados que aprovam a VC como potencial ferramenta para transferência de conhecimento. Os trabalhos empíricos em sua maioria concluem as experiências qualitativas com bons resultados. Os artigos teóricos concluem aprovando a visualização do conhecimento como potencial ferramenta para transferência de conhecimento.

Em comum, os autores relacionam a visualização do conhecimento às TICs. A VC parece carecer de pesquisas que impulsionem seu aperfeiçoamento. Não foram encontradas ferramentas que auxiliem sua utilização na $\mathrm{EaD}$, por exemplo.

\section{Conclusão}

O presente artigo visou analisar a técnica de VC como potencial ferramenta para auxilio no processo de ensino-aprendizagem. A análise dos artigos nos permitiu conhecer as aplicações desta ferramenta na área da educação, assim como os principais autores que abordam o tema.

A pesquisa não identificou modelos ou diretrizes para aplicação em objetos educacionais. Isso representa um caminho de pesquisa a ser preenchido e que merece atenção das áreas de Mídias e Conhecimento.Com um modelo poderíamos construir soluções a partir da relação entre o tipo de conhecimento a ser transmitido, tipo de representação visual a ser utilizado e o tipo de visão de mundo dos interessados. Esses elementos-chave configurariam a base deste sistema comunicacional.

$\mathrm{Na}$ área da educação a VC pode ser utilizada em contextos que objetivam melhorar a compreensão dos alunos e resolver o problema da sobrecarga cognitiva. Dessa forma, aproximam o conteúdo a ser transferido à visão de mundo dos aprendizes, facilitando a transferência de conhecimento. Portanto, a $16^{\circ}$ Ergodesign - Congresso Internacional de Ergonomia e Usabilidade de Interfaces Humano Tecnológica: Produto, Informações Ambientes Construídos e Transporte

$16^{\circ}$ USIHC - Congresso Internacional de Ergonomia e Usabilidade de Interfaces Humano Computador

CINAHPA | 2017 - Congresso Internacional de Ambientes Hipermídia para Aprendizagem.

técnica de $\mathrm{VC}$, a pesar de se mostrar uma ferramenta potencial, carece de métodos de aplicação.

Como toda pesquisa, existiram algumas limitações. A primeira delas foi o escopo de busca, reduzido a quatro bases de dados, Scielo, Capes, Web of Science, Scopus, nos dias 23, 24 e 25 de fevereiro. Os nove artigos analisados permitiram ter uma ideia de como a visualização do conhecimento vem sendo abordada e aplicada na área de educação. Porém, existe potencial em ter um avanço ainda maior no conhecimento sobre esse assunto, incorporando outras bases de dados nacionais ou internacionais.

\section{Referências Bibliográficas}

BURKHARD, R. A. Knowledge Visualization \{The Use of Complementary Visual Representations for the Transfer of Knowledge. A Model, a Framework, and Four New Approaches. Tese (Doutorado) $\mid$ Swiss Federal Institute of Technology Zurich, Switzerland, 2005.

Associação Brasileira de Educação a Distância. Censo EaD 2016. Disponível em < http://www.abed.org.br/site/pt/midiateca/censo ead/1395/2016/09/censoead.br___2015/2016>. Acessado em 17 de janeiro de 2017 .

DRESCH, A. et al. Design science research: método de pesquisa para avanço da ciência $e$ tecnologia. Porto Alegre: Bookman, 2014.

EPPLER, M. J. BURKHARD, R. A. Visual representations in knowledge management: framework and cases. Journal of Knowledge Management, 2007.

IEEE. Institute of Electrical and Electronics Engineers LTSC. Disponível em: $<>$. Acessado em 15 de fevereiro de 2017.

HARDEN, A.; GOUCH, D. Quality and relevance apprasial. An introduction to systematic reviews. London: Sage, 2012.

LAUREL, B. Computers as Theater. Reading, 


\section{$16^{\circ}$ \\ ERGODESIGN USIHC CINAHPA}

$16^{\circ}$ Ergodesign - Congresso Internacional de Ergonomia e Usabilidade de Interfaces Humano Tecnológica: Produto, Informações Ambientes Construídos e Transporte

$16^{\circ}$ USIHC - Congresso Internacional de Ergonomia e Usabilidade de Interfaces Humano Computador

CINAHPA | 2017 - Congresso Internacional de Ambientes Hipermídia para Aprendizagem.

MA: Addison-Wesley Publishing Company, 1993.

NETTO, M.; PERASSI, R.; FIALHO, F. A. P.

Estudos semióticos: análise perceptiva e ar terceiridade peirceana na obra "Jogos infantis" de Pieter Bruegel. Projética, Londrina, 2013.

TERGAN, S-O. KELLER, T. BURKHARD, R. Integrating knowledge and information: digital concept maps as a bridging technology. Palgrave Macmillan Ltd. 2006.. 